\title{
TWO RECENT IMPROVEMENTS IN THE STAINING OF SPIROCHETES IN NERVOUS TISSUE
}

GEORGE S. STEVENSON, M.D.

Assistant in Neuropathology, Psychiatric Institute, Ward's Island NEW YORK

Since 1916 several important contributions to the study of neurosyphilis have appeared in the German literature. These have been based in part on studies of spirochetes made possible by Jahnel's ${ }^{1}$ modification of the method of silver impregnation. Little, if any, reference has been made to these contributions in our American literature. This is probably due to the failure of the periodicals to reach the United States, and to failure or delay in indexing. It is hoped that by here presenting Jahnel's method it will be more accessible and better appreciated. Comment, such as is justified by a short acquaintance with, and use of, the method, will be made.

Similarly the recently published cover-glass method of Warthin and Starry ${ }^{2}$ will be referred to.

\section{JAHNEL'S METHOD}

The failure of foreign workers to obtain satisfactorily constant results with existing silver impregnation methods in nervous tissue led Jahnel to evolve his method. Our experience with the method in the laboratory of the Psychiatric Institute has been highly satisfactory. The technic, while requiring a long time, offers few pitfalls if the directions are followed. The results are not only very useful, but actually beautiful. With a subdued yellow to yellowish-brown background the spirochetes stand out in marked contrast. The staining of nerve fibers is so suppressed as to eliminate any confusion with spirochetes. Granulation, fraying and precipitate are negligible. While in our experience no old chemicals have been used, Jahnel warns against these. $\mathrm{He}$ offers an alternate developer, ${ }^{3}$ but owing to the success obtained by using the simpler one, we have not tried the other. No modification of the method has been found necessary. Fixation should be complete; our most beautiful preparations were made with tissue that had been in formaldehyd ten years. In step 1 , it is desirable to wash under sterile conditions.

1. Jahnel, F.: München. med. Wchnschr. 67:932 (Aug. 6) 1920.

2. Warthin, A. S., and Starry, A. C.: Second Improved Method for the Demonstration of Spirochaeta Pallida in the Tissues, J. A. M. A. 76:234 (Jan. 22) 1921.

3. Jahne1, F.: München. med. Wchnschr. 67:1263 (Oct. 29) 1920. 


\section{JAHNEL'S METHOD FOR STAINING SPIROCHETES IN THE BRAIN}

1. Wash out in water, in from one to three days, thin pieces from 2 to $4 \mathrm{~mm}$. fixed in formaldehyd or alcohol.

2. Place in pure pyridin one to three days.

3. Wash in many changes of water until the pyridin odor almost disappears during a period of from two to three days; this is important. Then allow the pieces to remain a "few days" (einige tage) in a 5 to 10 per cent. formaldehyd solution, U. S. P.

4. Place in water again. (The time in water this time is not stated; probably the washing should be thorough.)

5. Treat with uranium nitrate (Merck), 1 per cent. solution in distilled water, one-half to one hour in the incubator at $37 \mathrm{C}$. The use of glass wool (lead free) under the tissue helps the penetration but is not absolutely necessary. The purpose of the uranium nitrate is to prevent the coincident staining of other elements of the nervous tissue.

6. Wash out in distilled water for one day.

7. Allow to remain in 96 per cent. alcohol three to eight days.

8. Wash out in distilled water until the block sinks.

9. Place the tissue in a dark (amber) flask and treat it with a freshly prepared silver nitrate solution, 1.5 per cent., five to eight days in the oven at $37 \mathrm{C}$.

10. Decant the silver nitrate solution; wash the tissue slightly in water; then transfer it to a solution made up as follows:

$$
\begin{aligned}
& 4 \text { per cent. aqueous solution of pyrogallol............. } 95 \\
& \text { Formaldehyd solution, U. S. P....................... } 5
\end{aligned}
$$

Allow the tissue to remain in the solution one to two days.

A later note states that, especially with thicker pieces and fresher material, short washings in water after the silver nitrate treatment and the use of the pyrogallol-acetone-pyridin developer is to be recommended. This developer is made as follows:

4 per cent. pyrogallol.......................... 90

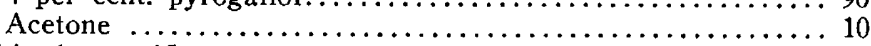

From this decant 15 c.c. and replace by pyridin.

11. Wash out in distilled water; embed in paraffin.

\section{COVER-GLASS METHOD}

The cover-glass method of Warthin and Starry, as originally published was not specifically adapted to nervous tissue. Treating the sections with uranium nitrate was given as one alternate in combating the failure of the tissue to stain properly. However, with nervous tissue, this treatment with uranium nitrate seems uniformly necessary. The cover-slip method offers two advantages: It is rapid, and it permits adjacent sections to be examined by other stains. On the other hand, it has the disadvantages of making the tissue somewhat granular, of staining the nerve fibers, although not seriously, and probably breaking up some of the spirochetes so that if few are in the section, the 
chance of finding them is reduced. Thus negative findings are inconclusive. After the paraffin mounts have been made (this may be done when cutting sections for the other stains), the method requires about one and one-half hours. From thirty minutes to one hour of this time is spent in impregnation, so that the work required actually takes less time. The main pitfall lies in developing. In preparing the developer it is necessary to work rapidly, to stir in the hydroquinone as quickly as possible, and to immerse the cover-slips edge up, since it is but a minute or two until the silver is reduced and the developer changed to an opaque mass. From this mass the cover-slips must be removed in order to determine whether the stain is sufficiently reduced. A yellowishbrown color is most desirable. In view of the fragility of the spirochete, it is further desirable to avoid too rapid hydration or dehydration. On this account we have suggested the graded alcohols in place of absolute alcohol.

\section{COVER-GLASS METHOD}

1. Fix completely, in 10 per cent. formaldehyd solution, U. S. P., pieces from 5 to $7 \mathrm{~mm}$. thick.

2. Embed in paraffin without washing in water.

3. Cut from 5 to 12 micron sections and mount on specially prepared coverslips with minimum albumin fixative (three-fourths inch No. 1 coverslips, cleaned thoroughly and dried in the incubator for twelve hours).

4. Remove the paraffin. (Warm over a flame, wash with xylol, descending alcohols and water.)

5. Immerse the section in 1 per cent. uranium nitrate (distilled water) five to eight minutes.

6. Wash in distilled water one minute.

7. Rinse the section and a clean cover-glass in fresh silver nitrate solution, 2 per cent. Apply the rinsed cover-glass to the section so that they will adhere by capillary attraction, and put edge up and against the side in a dark bottle containing half an inch $(1.27 \mathrm{~cm}$.) of fresh 2 per cent. silver nitrate solution; cork tightly. Incubate from thirty to sixty minutes in the dark.

8. Separate the cover-slips and put the section into the following reducer. Remove when yellowish to reddish brown.

Mix 2 per cent. silver nitrate 3 c.c., warm 10 per cent. aqueous gelatin 5 c.c., warm glycerin 5 c.c.

With stirring add warm 1.5 per cent. agar suspension, 5 c.c. (The agar suspension is made by mixing $1.5 \mathrm{gm}$. agar with 100 c.c. cold distilled water, heating slowly, and stirring constantly until it boils. .Continue to boil until suspension is complete. Keep on top the paraffin oven.)

With stirring add a 5 per cent. aqueous hydroquinone solution 1 to 2 c.c. just before immersing sections.

9. Rinse the section in a 5 per cent. solution of sodium thiosulphate.

10. Rinse the section in distilled water.

11. Treat with ascending alcohols, xylol; mount it in balsam. 